https://helda.helsinki.fi

\title{
Low childhood high density lipoprotein cholesterol levels and subsequent risk for chronic inflammatory bowel disease
}

\section{Voutilainen, Markku}

2018-04

Voutilainen , M , Hutri-Kähönen , N , Tossavainen , P , Sipponen , T , Pitkänen , N , Laitinen , T , Jokinen , E , Rönnemaa , T , Viikari , J S A, Raitakari , O T \& Juonala , M 2018 , ' Low childhood high density lipoprotein cholesterol levels and subsequent risk for chronic inflammatory bowel disease ' , Digestive and Liver Disease , vol. 50 , no. 4 , pp. 348-352 . https://doi.org/10.1016/j.d

http://hdl.handle.net/10138/301297

https://doi.org/10.1016/j.dld.2018.01.121

publishedVersion

Downloaded from Helda, University of Helsinki institutional repository.

This is an electronic reprint of the original article.

This reprint may differ from the original in pagination and typographic detail.

Please cite the original version. 
Alimentary Tract

\title{
Low childhood high density lipoprotein cholesterol levels and subsequent risk for chronic inflammatory bowel disease
}

\author{
Markku Voutilainen $^{\mathrm{a}, \mathrm{b}, *}$, Nina Hutri-Kähönen ${ }^{\mathrm{c}}$, Päivi Tossavainen ${ }^{\mathrm{d}}$, Taina Sipponen ${ }^{\mathrm{e}}$, \\ Niina Pitkänen ${ }^{\mathrm{f}}$, Tomi Laitinen ${ }^{\mathrm{g}}$, Eero Jokinen ${ }^{\mathrm{h}}$, Tapani Rönnemaa ${ }^{\mathrm{a}}{ }^{\mathrm{a}}$, Jorma S.A. Viikari ${ }^{\mathrm{a}} \mathrm{b}$ \\ , Olli T. Raitakari ${ }^{\mathrm{b}, \mathrm{f}}$, Markus Juonala ${ }^{\mathrm{a}, \mathrm{b}}$ \\ a Department of Medicine, University of Turku, Turku, Finland \\ b Division of Medicine, Turku University Hospital, Turku, Finland \\ ${ }^{\mathrm{c}}$ Department of Pediatrics, University of Tampere and Tampere University Hospital, Tampere, Finland \\ d Department of Pediatrics, PEDEGO Research Unit and Medical Research Center, Oulu University Hospital and University of Oulu, Oulu, Finland \\ e Department of Medicine, Division of Gastroenterology, Helsinki University Hospital, Helsinki, Finland \\ ${ }^{\mathrm{f}}$ Research Centre of Applied and Preventive Cardiovascular Medicine; University of Turku, Turku, Finland \\ ${ }^{g}$ Department of Clinical Physiology and Nuclear Medicine, Kuopio University Hospital and University of Eastern Finland, Kuopio, Finland \\ h Department of Pediatric Cardiology, Hospital for Children and Adolescents, University of Helsinki, Helsinki, Finland
}

\section{A R T I C L E I N F O}

\section{Article history:}

Received 21 September 2017

Accepted 6 January 2018

Available online 31 January 2018

\section{Keywords:}

Crohn's disease

Genetics

High density lipoprotein cholesterol

Inflammatory bowel disease

Ulcerative colitis

\begin{abstract}
A B S T R A C T
Background and aims: Several genetic and environmental risk factors have been linked to chronic inflammatory bowel disease (IBD). The incidence of IBD has significantly increased in developed countries during last decades. The aim of the present study was to examine childhood risk factors for subsequent IBD diagnosis in a longitudinal cohort study of children and adolescents.

Methods: A Finnish study population consisting of 3551 children and adolescents originally evaluated as part of the Cardiovascular Risk in Young Finns study in 1980. At baseline, participant BMI, insulin, lipid, C-reactive protein and blood pressure levels, socioeconomic position, dietary habits, and physical activity, were evaluated. In addition, information was gathered on rural residency, severe infections, breast feeding, parental smoking and birth weight. Subsequent IBD diagnosis status was evaluated based on nationwide registries on hospitalisations and drug imbursement decisions.

Results: Altogether, 49 participants (1.4\%) had IBD diagnosed during the 34 years of register follow-up, of which 31 had ulcerative colitis, 12 Crohn's disease and 6 undetermined colitis. In univariate analyses, significant correlations were observed between childhood HDL-cholesterol (risk ratio (95\% CI) for 1-SD change (0.58 (0.42-0.79)) and CRP concentrations (1.20 (1.01-1.43)) with IBD. The inverse association between HDL-cholesterol and IBD remained significant $(0.57(0.39-0.82))$ in a multivariable model including data on age, sex and CRP. In addition, a weighted genetic $z$-score of 71 single nucleotide polymorphisms associated with elevated HDL-cholesterol levels was significantly lower in IBD patients, $P=0.01$ ).

Conclusion: Low childhood HDL-cholesterol levels are associated with subsequent IBD diagnosis. In addition, a genetic risk score associated with low HDL-cholesterol levels predict later IBD suggesting that HDL-cholesterol metabolism might have a role in the pathogenesis of IBD.
\end{abstract}

(C) 2018 Editrice Gastroenterologica Italiana S.r.l. Published by Elsevier Ltd. All rights reserved.

\section{Introduction}

In developed countries, the incidence of chronic inflammatory bowel disease (IBD), i.e. ulcerative colitis and Crohn's disease, has

\footnotetext{
* Corresponding author at: Division of Medicine, Turku University Hospital, Kiinamyllynkatu 4-8, FIN-20520 Turku, Finland.

E-mail address: markku.voutilainen@tyks.fi (M. Voutilainen).
}

increased more than 10 -fold during last decades [1]. IBD is incurable and $80 \%$ of patients with Crohn's disease need surgical therapy for the complications of the disease. Among children, the incidence of IBD has increased more than 3-fold in Finland during the last three decades and the increase has been most pronounced among adolescents [2]. In children aged less than 10 years, the increase has been lower suggesting possible environmental triggers for IBD [2]. The incidence of paediatric IBD in Finland is comparable to other developed countries [3]. Within Finland, a geographical variation 
in the prevalence of ulcerative colitis, but not in Crohn's disease, has been reported with a higher prevalence in the northern parts of the country [4], and the incidence of paediatric IBD has been shown to be higher in areas with a low density of child population [5]. In other countries, a low environmental hygiene level has been associated with lower IBD prevalence [6].

In addition to environmental risk factors, genetic factors contribute to the risk of IBD. Family members of patients with Crohn's disease have an increased risk. Compared to dizygotic twins, monozygotic twins have a significantly higher risk of Crohn's disease [7]. Childhood-onset Crohn's disease has more often aggressive phenotype [8]. Although more than 160 genetic loci are associated with the risk of developing IBD, the known risk alleles may explain only a quarter of the heritability of Crohn's disease [9].

At present, there is a lack of prospective data concerning childhood risk factors for subsequent IBD. Using data from the longitudinal Cardiovascular Risk in Young Finns Study cohort, our aim was to examine the association between possible childhood (age 3-18 years) risk factors and subsequent IBD occurrence during 34 years of follow-up among 3551 individuals. These data could provide novel information on childhood exposures predicting later IBD.

\section{Methods}

\subsection{Participants}

The Cardiovascular Risk in Young Finns Study is an ongoing prospective study focusing cardiovascular risk factors from childhood to adulthood. The baseline examination was in 1980, when participants were aged 3-18 years. The latest clinical examination was performed in 2011. In addition, these individuals have been followed up through national registries including data on clinical diagnoses. The current sample included 3551 individuals with baseline risk exposure data. The study complies with the Declaration of Helsinki and has institutional ethics approval. Written informed consent was obtained from all participants.

\subsection{IBD diagnoses data}

Diagnose codes for Crohn's disease (ICD-9 diagnoses 555.X and ICD-10 diagnoses $\mathrm{K} 50 . \mathrm{X}$ ) and ulcerative colitis (ICD-9 diagnoses 556.X and ICD-10 diagnoses K51.X) were extracted from the Finnish national hospitalization database (which commenced in 1969). In addition, similar data extraction was performed from the Finnish Social Insurance Institution's drug imbursement register. Since 1986, the Social Insurance Institution has recorded all decisions on the entitlements for the special refunds of IBD patients in a nationwide register. Between 1986 and 1993, CD and UC had separate reimbursement codes in the register. Between 1994 and 1999, CD and UC were recorded with the same IBD-code, and diagnosis codes of subtypes were not registered. Therefore the prevalence of CD and UC cannot be calculated separately between 1994 and 1999. Since 2000, the ICD-10-codes K50 and K51 have been available to separately identify patients with $\mathrm{CD}$ and patients with UC.

\subsubsection{Physical variables}

Height and weight, rounded to the nearest $0.5 \mathrm{~cm}$ and $0.1 \mathrm{~kg}$ respectively, were measured at all time-points using standardized protocols, and BMI was calculated as weight $(\mathrm{kg})$ divided by height (m) squared. Baseline blood pressure at three years of age was measured by an ultrasound device and at other childhood ages by a mercury sphygmomanometer. A random zero sphygmomanometer was used in adults. The first and fifth Korotkoff sounds were used to define systolic and diastolic blood pressures, which were averaged from three measurements.

\subsubsection{Laboratory variables}

Blood samples were obtained following a 12-h fast. Standard enzymatic methods were used for serum total cholesterol, triglycerides, high-density lipoprotein (HDL) cholesterol, and plasma glucose. HDL cholesterol was measured after dextran sulfate precipitation and low-density lipoprotein (LDL) cholesterol was calculated using the Friedewald formula. High sensitivity Creactive protein (hsCRP) was measured by an automated analyzer using a latex turbidimetric immunoassay. For hsCRP analyses, childhood serum samples were taken in 1980 and stored in $-20^{\circ} \mathrm{C}$. These samples were analyzed in 2005. During the storage, the samples were not thawed or refrozen.

\subsubsection{Anamnestic variables}

In childhood, questionnaires completed by the participant or their parent were used to obtain data on physical activity, birth weight, family income, rural/urban residence, breast feeding in infancy, fruit and vegetable consumption, and parental smoking. Physical activity was assessed with questions concerning the frequency and intensity of physical activity and a physical activity index was calculated based on the variables as previously described [10]. There were two different kinds of physical activity questionnaires for the younger (three to six year olds, a parent completed questionnaire) and older children (9-18 year olds, self-completed questionnaire). The calculated physical activity indices were age-standardized to allow comparison across age groups. Annual family income strata at the time of enrolment was determined as follows: [category 1] $<12,500$ Finnish marks (FIM) ( 5850 EUR); [2] 15,001-25,000 FIM; [3] 25,001-35,000 FIM; [4] 35,001-45,000 FIM; [5] 45,001-55,000 FIM; [6] 55,001-75,000 FIM; [7] 75,001-100,000 FIM; [8] >100.000 FIM.

\subsubsection{Hospital discharge data on infections}

Infection-related hospitalization was defined as a hospital discharge diagnosis that included at least one International Classification of Disease (ICD) infection-related code as either a primary or secondary code. Hospitalization was defined as an admission that included at least one overnight stay. We used both primary and secondary codes to ensure the capture of all infections, an approach we and others have used previously [11].

\subsection{Genotyping and construction of the genetic risk score}

Genotyping was performed using the Illumina Human $670 \mathrm{~K}$ Bead Chip, and imputation was performed using SHAPEIT v1 [12] and IMPUTE2 [13] software and the 1000G Phase I Integrated Release Version 3 as a reference panel [14].

\subsection{Statistical analyses}

Baseline (in 1980) risk factor levels between individuals with and without IBD diagnoses during the follow-up were compared by t-test for continuous and Chi-square test for categorical variables. Thereafter, those factors significantly associated with IBD were included in a multivariable logistic regression model. This model also included age and sex. In addition, among those factors with significant association for IBD, sensitivity analyses were performed separately for males and females, as well as for both IBD outcomes, i.e. ulcerative colitis and Crohn's disease.

A weighted genetic risk score comprising 71 SNPs associated with HDL-C in a recent large GWAS [15] was calculated as a sum of the genotyped risk alleles or imputed allele dosages carried by an individual each multiplied by the externally reported effect size. 
Table 1

Baseline (in 1980) characteristics.

\begin{tabular}{llll}
\hline Variable & $\begin{array}{l}\text { No IBD } \\
\text { during } \\
\text { follow-up }\end{array}$ & $\begin{array}{l}\text { IBD during } \\
\text { follow-up }\end{array}$ & P-value \\
\hline$N$ & 3502 & 49 & \\
Age (years) & $10.5 \pm 5.0$ & $10.7 \pm 5.2$ & 0.84 \\
Males (\%) & 49.1 & 52.0 & 0.75 \\
BMI (kg/m ${ }^{2}$ ) & $17.9 \pm 3.1$ & $17.8 \pm 2.8$ & 0.91 \\
Systolic BP (mmHg) & $113 \pm 12$ & $113 \pm 12$ & 0.96 \\
LDL-cholesterol (mmol/l) & $3.43 \pm 0.83$ & $3.30 \pm 0.82$ & 0.28 \\
HDL-cholesterol (mmol/l) & $1.56 \pm 0.31$ & $1.41 \pm 0.26$ & 0.0006 \\
Triglycerides (mmol/l) & $0.66 \pm 0.31$ & $0.69 \pm 0.27$ & 0.58 \\
Insulin (IU/l) & $9.6 \pm 6.0$ & $9.1 \pm 4.9$ & 0.61 \\
CRP (mg/l) & $1.0 \pm 3.0$ & $2.0 \pm 4.4$ & 0.04 \\
Fruit consumption (servings/week) & $6.9 \pm 2.8$ & $7.3 \pm 2.6$ & 0.34 \\
Vegetable consumption & $6.3 \pm 2.9$ & $6.4 \pm 2.9$ & 0.78 \\
(servings/week) & & & \\
Physical activity index & $10.9 \pm 3.7$ & $10.6 \pm 3.3$ & 0.61 \\
Birth weight (kg) & $3.51 \pm 0.55$ & $3.41 \pm 0.59$ & 0.25 \\
Breast feeding (\%) & 85.3 & 85.0 & 0.97 \\
Rural resident (\%) & 47.2 & 52.0 & 0.42 \\
Family income (categorical variable & $4.8 \pm 1.9$ & $4.7 \pm 2.2$ & 0.65 \\
1-8) & & & \\
Parental smoking (\%) & 46.5 & 55.6 & 0.17 \\
Childhood infection related & 7.6 & 10.0 & 0.47 \\
hospitalisations (\%) & & & \\
\hline
\end{tabular}

Plus-minus values are mean \pm SD. $P$-values from $t$-test for continuous and Chisquare test for categorical variables.

Table 2

Prevalence of inflammatory bowel disease.

\begin{tabular}{lll}
\hline Disease & Females & Males \\
\hline Inflammatory bowel disease & $26(1.4 \%)$ & $23(1.3 \%)$ \\
Ulcerative colitis & $16(0.9 \%)$ & $15(0.9 \%)$ \\
Crohn disease & $8(0.5 \%)$ & $4(0.2 \%)$ \\
Unspecific colitis & $2(0.1 \%)$ & $4(0.2 \%)$ \\
\hline
\end{tabular}

The weighted genetic risk score was standardized according to the mean and SD before the analyses. Comparison of genetic risk score values between individuals with and without IBD was performed with $t$-test.

Statistical analyses were performed using $\operatorname{SAS}^{\circledR} 9.3$ or in the case of the genetic analyses with R software version 3.3.2.

\section{Results}

\subsection{Characteristics of the study cohort}

Baseline characteristics are shown in Table 1. At the beginning of the study in 1980, 3551 persons were included, of which 1805 (50.8\%) were female. At the end of the follow-up, 49 persons (1.4\%) had IBD diagnosed, of those 26 were female (Table 2). Of the IBD patients, 31 had ulcerative colitis, 12 Crohn's disease and 6 had undetermined colitis. Sex ratios (M:F) were 1:1.1, 1:2 and 2:1 for ulcerative colitis, Crohn's disease and undetermined colitis, respectively.

\subsection{Childhood risk factors predicting subsequent IBD}

Univariate age and sex adjusted comparisons between IBD diagnosis, and demographic, clinical and laboratory variables revealed statistically significant inverse correlation between HDLcholesterol and CRP concentrations with IBD (Table 3). In a multivariable analysis including HDL-cholesterol concentration, C-reactive protein concentration, age, sex an inverse association childhood HDL-cholesterol and subsequent IBD diagnosis remained statistically significant (OR for 1-SD change in HDL-cholesterol 0.57 ; $95 \%$ confidence interval $0.39-0.82, P=0.006$ ) (Table 4 ).
Table 3

Univariate associations between childhood factors (in 1980) and later IBD.

\begin{tabular}{ll}
\hline Variable & OR $(95 \% \mathrm{CI})$ \\
\hline BMI & $0.94(0.64-1.38)$ \\
Systolic BP & $1.00(0.72-1.39)$ \\
LDL-cholesterol & $0.84(0.62-1.14)$ \\
HDL-cholesterol & $0.58(0.42-0.79)$ \\
Triglycerides & $1.07(0.82-1.40)$ \\
Insulin & $0.87(0.60-1.25)$ \\
CRP & $1.20(1.01-1.43)$ \\
Fruit consumption & $1.19(0.87-1.61)$ \\
Vegetable consumption & $1.02(0.76-1.36)$ \\
Physical activity index & $1.03(0.85-1.25)$ \\
Birth weight & $0.84(0.62-1.13)$ \\
Breast feeding & $0.96(0.40-2.33)$ \\
Rural resident & $1.21(0.69-2.12)$ \\
Family income & $0.95(0.72-1.26)$ \\
Parental smoking & $1.43(0.79-2.60)$ \\
Childhood infection related hospitalisations & $1.39(0.53-3.65)$ \\
\hline
\end{tabular}

OR-values are for 1-SD change in continuous variables. All analyses are adjusted with age and sex.

Table 4

Multivariate model of childhood predictors of IBD.

\begin{tabular}{ll}
\hline Variable & OR $(95 \% \mathrm{CI})$ \\
\hline HDL-cholesterol & $0.57(0.39-0.82)$ \\
CRP & $1.16(0.96-1.41)$ \\
Age & $1.02(0.96-1.09)$ \\
Male sex & $1.00(0.52-1.93)$ \\
\hline
\end{tabular}

OR-values are from logistic regression model for 1-SD change in continuous variables.

Table 5

Sensitivity analyses for the association between childhood HDL-cholesterol and later IBD in different subgroups.

\begin{tabular}{ll}
\hline Analysis group & OR $(95 \% \mathrm{CI})$ \\
\hline Females & $0.56(0.36-0.86)$ \\
Males & $0.59(0.38-0.93)$ \\
Outcome: ulcerative colitis & $0.63(0.43-0.92)$ \\
Outcome: Crohn disease & $0.66(0.36-1.22)$ \\
\hline
\end{tabular}

OR-values are from logistic regression model for 1-SD change in continuous variables. All analyses are adjusted with age, outcome-specific analyses are also adjusted with sex.

HDL-cholesterol levels in adulthood (in 2011) were lower among IBD patients compared to the non-IBD individuals $(1.22 \pm 0.32$ vs. $1.32 \pm 0.32 \mathrm{mmol} / \mathrm{l}, P=0.02)$. When the results shown in Table 4 were additionally adjusted with adulthood HDL-cholesterol level (in 2011), the association between childhood HDL-cholesterol and subsequent IBD remained significant $(P=0.003)$.

As shown in Table 5, in sex-specific analyses, the association between childhood HDL-cholesterol and IBD was significant in both genders. In outcome-specific analyses, the association was significant for ulcerative colitis.

\subsection{Genetic analyses between IBD and HDL-cholesterol associated SNPS}

Compared with persons without IBD, weighted genetic $z$-score of 71 single nucleotide polymorphism (SNP) associated with HDL cholesterol was significantly lower in IBD patients (Fig. 1).

\section{Discussion}

In the present prospective cohort study, we observed a significant inverse correlation between childhood HDL-cholesterol levels and subsequent IBD diagnosis. This finding was further confirmed 


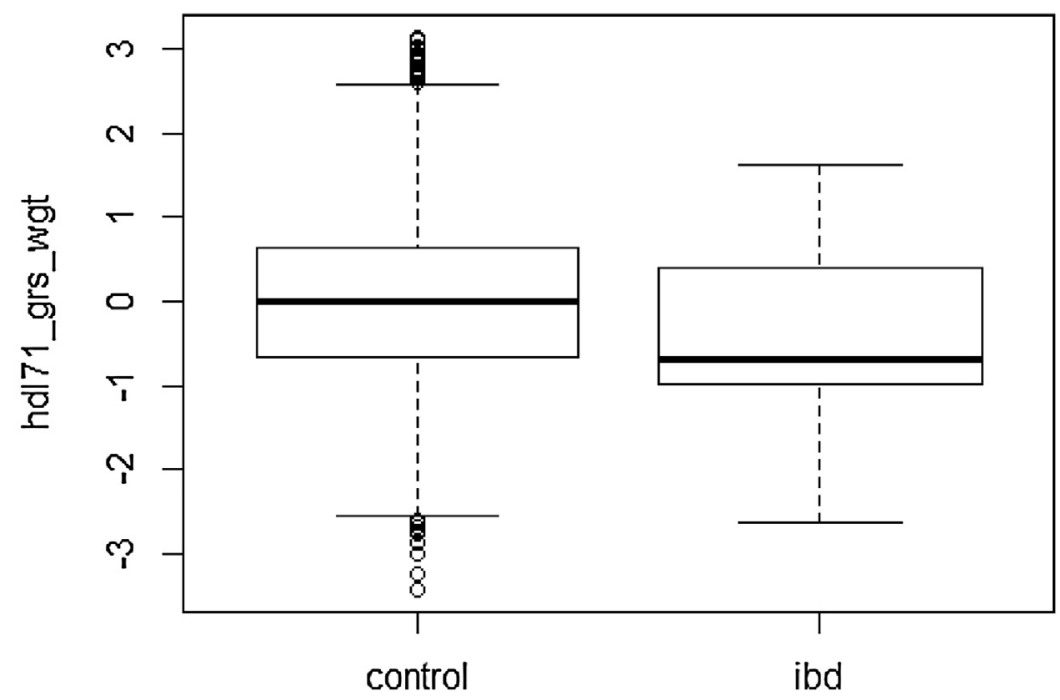

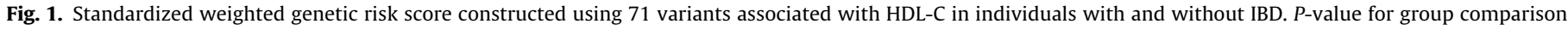
0.01 .

by the observation that a genetically derived SNPs related to higher HDL-cholesterol levels were detected less frequently among individuals with IBD diagnosis. To our knowledge, this is the first observation linking low genetically determined childhood HDLcholesterol level to increased IBD risk.

There is a paucity of data concerning the association between early life HDL-cholesterol levels and later risk of IBD. In contrast to our findings based on a longitudinal population-based cohort study of over 3500 children, a smaller, cross-sectional Polish study reported similar lipid profiles in 30 children with first IBD flare and 20 healthy controls [16]. Among young adults (mean age 31 years), Cappello et al. observed that total and LDL-cholesterol levels were statistically significantly lower in IBD patients compared to controls whereas the difference was non-significant in HDL-cholesterol levels [17]. Low HDL-cholesterol level is an established cardiovascular risk factor and previous studies have found an increased risk for coronary artery disease (CAD) in adult IBD patients. In Finnish studies, it has been observed that cardiovascular mortality risk is higher among IBD patients [18,19]. These results are concordant with Canadian and Danish studies that detected an increased coronary artery disease risk in IBD patient cohorts of over 8000 and 28,000 patients, respectively [20,21]. A study from Denmark revealed that active IBD worsened patient's prognosis after myocardial infarction [22]. However, the above-mentioned findings were not confirmed in UK and US studies [23,24]. The pathogenesis of atherosclerotic disease and the role of conventional cardiovascular risk factors, such as low HDL-cholesterol levels, are still not completely understood among IBD patients. It is noteworthy, that in addition to traditional CAD risk factors, IBD patients have also nonconventional risk factors such as inflammatory activity of IBD and the use of disease-modifying therapy that could modify CAD morbidity and mortality [25].

The treatment of adverse lipid levels may have impact on the risk and course of IBD. The use of statins significantly decreases the risk on both new onset ulcerative colitis and Crohn's disease [26]. Statin use is also associated with reduced use of steroids in ulcerative colitis [27]. The impact of statins on IBD may be due to their anti-inflammatory effect, because they also attenuate disease activity in rheumatoid arthritis [28] and systemic lupus erythematosus [29]. Present results suggest that increased emphasis on HDL levels already in childhood might be beneficial.

The pathogenesis of IBD is complex. An immunological dysfunction with primary and secondary inflammatory reactions is the hallmark of IBD, and genetic and environmental factors as well as gut microbiota are involved [30]. Results of a large international study suggest that genetic factors determine mainly disease location and type (ulcerative colitis, Crohn's colitis and ileal Crohn's disease), but environmental factors such as diet, smoking and microbiota may be even stronger contributors to IBD phenotypes [31]. Lipids and lipoproteins may have impact on the risk of IBD and its course. HDL-cholesterol and its main apolipoprotein apoA-I reduce cholesterol in lipid rafts of several immune cell types leading to the modulation of key receptors involved in both innate and adaptive immune responses [32]. HDL has anti-inflammatory effects as a part of innate immune system and low levels of HDL cholesterol are associated with inflammatory and immune diseases [33]. Furthermore, inflammation also reportedly decreases HDLcholesterol levels and induces changes in HDL-associated proteins possibly leading to an increased risk of atherosclerosis [34].

The strength of the present study is its study population representing a randomly selected ethnically homogeneous young age cohort with large data on socioeconomic, clinical and laboratory factors. Furthermore, the follow-up time was over thirty years and well into middle adulthood of our participants. As IBD is most commonly diagnosed in adolescence and early adulthood [35], we are likely to have covered most cases in our sample.

A major limitation in the present study is the low number of diagnosed IBD cases with different IBD types possibly resulting in selection bias. IBD diagnoses were not acquired by clinical followup, but were derived from national registries. Therefore, possible misclassification of non-IBD cases might have occurred. However, earlier Finnish reports have shown that the completeness and accuracy in Finnish registers are very good, e.g. positive predictive value for gastrointestinal diseases is over $90 \%$ in the hospital discharge register [36]. The accuracy of the drug imbursement data is also high. A study group evaluated 50 randomly selected IBD reimbursement reports to assess consistency in diagnostic criteria. Only one of the 50 diagnoses was questionable [37]. According to a Finnish survey, IBD patients have been diagnosed by specialist (in Finland usually a gastroenterologist or a specialist in internal medicine) in over $96 \%$ of cases [38]. Moreover, the combined IBD incidence rates $(C D+U C)$ in our cohort are in line with prior reports in Finland [4].

Another limitation is that the age of patients at the time of IBD diagnosis was not available. As the analyses were derived from a cardiovascular cohort study initiated in 1980, we were not able to include all possible risk factors for subsequent IBD in our 
analyses. Furthermore, the present results are generalizable only to Caucasian populations. Finally, we were not able to perform replication analyses in another cohort to confirm our findings. Further studies are required to investigate the association between HDL-cholesterol and IBD.

In conclusion, the present study revealed that low childhood HDL-cholesterol levels associate with the subsequent risk of clinical IBD. Moreover, a genetic risk score related with elevated HDL-cholesterol levels was lower among IBD patients. These data suggest that low HDL-cholesterol level is a possible risk factor for IBD and therefore warrant further research to explore the mechanisms explaining this association to possibly provide novel therapeutic options. From a clinical point of view, it is important to evaluate whether HDL-cholesterol data can improve IBD risk prediction.

\section{Conflict of interest}

None declared.

\section{Funding}

The Young Finns study has been financially supported by the Academy of Finland (grants 126925, 121584, 124282, 129378, 117787 and 41071), the Social Insurance Institution of Finland; Kuopio, Tampere, and Turku University Hospital Medical Funds, Juho Vainio Foundation; Paavo Nurmi Foundation, Finnish Foundation of Cardiovascular Research, TYKS Foundation, Finnish Cultural Foundation, Sigrid Juselius Foundation, and Yrjö Jahnsson Foundation.

\section{References}

[1] Cosnes J, Gower-Rousseau C, Seksik P, et al. Epidemiology and natural history of inflammatory bowel disease. Gastroenterology 2011;140:1785-94.

[2] Virta L, Saarinen M, Kolho K-L. Inflammatory bowel disease incidence is on the continuous rise among all pediatric patients except for the very young: a nationwide registry-based study on 28-year follow-up. J Crohn's Colitis 2017; 11:152-6.

[3] Müller KE, Lakatos PL, Arató A, et al. Hungarian IBD Registry Group (HUPIR), Incidence, Paris classification, and follow-up in a natiowide incident cohort of pediatric patients with inflammatory bowel disease. J Pediatr Gastroenterol Nutr 2013;57(5):576-82.

[4] Jussila A, Virta L, Salomaa V, et al. High and increasing prevalence of inflammatory bowel disease in Finland with a clear North-South difference. J Crohn's Colitis 2013;7:e256-62.

[5] Lehtinen P, Pasanen K, Kolho K-L, et al. Incidence of pediatric inflammatory bowel disease in Finland: an environmental study. JPGN 2016;63:65-70.

[6] Cholapranee A, Ananthakrishnan A. Environmental hygiene and risk of inflammatory bowel diseases: a systematic review and meta-analysis. Inflamm Bowel Dis 2016;22:2191-9.

[7] Loddo I, Romano C. Inflammatory bowel disease: genetics, epigenetics, and pathogenesis. Front Immunol 2015 , http://dx.doi.org/10.3389/fimmu 2015.00551.

[8] Duricova D, Fumery M, Annese V, et al. The natural history of Crohn's disease in children: a review of population-based studies. Eur J Gastroenterol Hepatol 2017;29:125-34.

[9] Moran C, Klein C, Muise A, et al. Very early-onset inflammmatory bowel disease: gaining insight through focused discovery. Inflamm Bowel Dis 2015:21:1166-75.

[10] Telama R, Viikari J, Välimäki I, et al. Atherosclerosis precursors in Finnish children and adolescents. X. Leisure-time physical activity. Acta Ped Scand Auppl 1985;318:169-80.

[11] Burgner D, Savin M, Magnussen C, et al. Infection-related hospitalization in childhood and adult metabolic outcomes. Pediatrics 2015;136:e554-62.
[12] Delaneau O, Marchini J, Zagury J. A linear complexity phasing method for thousands of genomes. Nat Methods 2011;9:179-81.

[13] Howie B, Donnelly P, Marchini J. A flexible and accurate genotype imputation method for the next generation of genome-wide association studies. PLoS Genet 2009;5:e1000529.

[14] 1000 Genomes Project Consortium, Abecasis G, Altshuler D, et al. A map of humn genome variation from population-scale sequencing. Nature 2010;467:1061-73.

[15] Global Lipids Genetics Consortium, Willer C, Schmidt E, et al. Discovery and refinement of loci associated with lipid levels. Nat Genet 2013;45:1274-83.

[16] Pac-Koźuchowska E, Krawiec P, Mrozkowska-Juchkiewicz A, et al. Inflammatory and lipid-associated markers of cardiovascular diseases in children with first exacerbation of inflammatory bowel disease. Med Sci Monit 2016;22:164334-9.

[17] Cappello M, Licata A, Calvaruso V, et al. Increased expression of markers of early atherosclerosis in patients with inflammatory bowel disease. Eur J Intern Med 2017;37:83-9.

[18] Haapamäki J, Roine R, Turunen U, et al. Increased risk for coronary heart disease, asthma, and connective tissue diseases in inflammatory bowel disease. Crohn's Colitis 2011;5:41-7.

[19] Jussila A, Virta L, Pukkala E, et al. Mortality and causes of death in patients with inflammatory bowel disease: a nationwide register study in Finland. J Crohn's Colitis 2014;8:1088-96.

[20] Bernstain C, Wajda A, Blanchard J. The incidence of arterial thromboembolic diseases in inflammatory bowel disease: a population-based study. Clin Gastroenterol Hepatol 2008;6:41-5.

[21] Rungoe C, Basit S, Ranthe M, et al. Risk of ischaemic heart disease in patients with inflammatory bowel disease: a nationwide Danish cohort study. Gut 2013;62:689-94.

[22] Kristensen S, Ahlehoff O, Lindhardsen J, et al. Prognosis after first-time myocardial infarction in patients with inflammatory bowel disease according to disease activity. Circ Cardiovasc Qual Outcomes 2014;7:857-62.

[23] Osterman M, Yang Y, Brensinger C, et al. No increased risk of myocardial infarction among patients with ulcerative colitis or Crohn's disease. Clin Gastroenterol Hepatol 2011;9:875-80

[24] Barnes E, Beery R, Schulman A, et al. Hospitalizations for acute myocardia infarction are decreased among patients with inflammatory bowel disease using a nationwide inpatient database. Inflamm Bowel Dis 2016;22:2229-37.

[25] Singh S, Kullo I, Pardi D, et al. Epidemiology, risk factors and management of cardiovascular diseases in IBD. Nat Rev Gastroenterol Hepatol 2015;12:26-35.

[26] Ungaro $R$, Chang $H$, Cote-Daigneaut J, et al. Statins associated with decreased risk of new onset inflammatory bowel disease. Am J Gastroenterol 2016;111:1416-23.

[27] Crockett S, Hansen R, Stürmer T, et al. Statins are associated with reduced use of steroids in inflammatory bowel disease: a retrospective cohort study. Inflamm Bowel Dis 2012;18:1048-56.

[28] Lv S, Liu Y, Zou Z, et al. The impact of statins therapy on disease activity and inflammatory factor in patients with rheumatoid arthritis: a meta-analysis. Clin Exp Rheumatol 2015;33:69-76.

[29] Fatemi A, Moosavi M, Sayedbonakdar Z, et al. Atorvastatin effect on systemic lupus erythematosus disease activity: a double-blind randomized clinical trial. Clin Rheumatol 2014;33:1273-8.

[30] de Souza H, Fiocchi C. Immunopathogenesis of IBD: current state of the art. Nat Rev Gastroenterol Hepatol 2016;13:13-20.

[31] Cleynen I, Boucher G, Jostins L, et al. Inherited determinants of Crohn's disease and ulcerative colitis phenotypes: a genetic association study. Lancet 2016;387:156-67.

[32] Catapano A, Pirillo A, Bonacina F, et al. HDL in innate and adaptive immunity. Cardiovasc Res 2014;103:372-83.

[33] Yu B, Wang S, Peng D, et al. HDL and immunomodulation: an emerging role of HDL against atherosclerosis. Immunol Cell Biol 2010;88:285-90.

[34] Feingold K, Grunfeld C. Effect of inflammation on HDL stucture and function. Curr Opin Lipidol 2016;27:521-30.

[35] Kaplan G. The global burden of IBD: from 2015 to 2025. Nat Rev Gastroenterol Hepatol 2015;12:720-7.

[36] Sund R. Quality of the Finnish Hospital Discharge Register: a systematic review. Scand J Public Health 2012;40:505-15.

[37] Lehtinen P, Ashorn M, Iltanen S, et al. Incidence trends of pediatric inflammatory bowel disease in Finland, 1987-2003, a nationwide study. Inflamm Bowel Dis 2011;8:1778-83.

[38] Haapamäki J, Turunen U, Roine RP, et al. Finnish patients with inflammatory bowel disease have fewer symptoms and are more satisfied with their treatment than patients in the previous European study. Scand J Gastroenterol 2008; $7: 821-30$. 